\title{
A catalog of the types of Gyrinidae, Halliplidae, Noteridae, Dytiscidae, Limnichidae, Elmidae, Dryopidae, Heteroceridae and Scirtidae (Insecta, Coleoptera) deposited in the Museo Argentino de Ciencias Naturales, Buenos Aires
}

\author{
Axel O. BACHMANN \\ Entomology Division, Museo Argentino de Ciencias Naturales “Bernardino Rivadavia», Av. A. Gallardo 470, \\ 1405 Buenos Aires, Argentina. Researcher of CONICET
}

\begin{abstract}
The type specimens (all current categories) of Gyrinidae, Haliplidae, Dytiscidae, Noteridae (Adephaga), Limnichidae, Elmidae, Dryopidae, Heteroceridae and Scirtidae (Polyphaga) deposited in this Museum are listed; 52 names are recorded, 31 of them (60\%) represented here by name-bearing types ('primary' types). 'The specific and subspecific names were alphabetically filed, followed by the generic ones as they were spelled in the original publication (or the generic and specific names in the case of subspecies and varieties). Later combinations and/or current binomina are mentioned, as far as these are known to the author. Two lists are added: 1. of specimens labelled as types of names not found in the literature, and presumably not published, and 2 . of specimens labelled as types, but not originally included as such, and published or not after the original description.
\end{abstract}

Key words: water beetles, type specimens, museum collection, Argentina.

A list of the type specimens of the species of aquatic coleopteran families (exclusive of Hydrophiloidea) deposited in the Entomological Division of this Museum is presented, corresponding to 52 names: 10 holotypes, 32 syntypes (of 21 names) 1 of them dubious, 130 paratypes (includ. ing 5 allotypes), and 1 paralectotype; 31 of these 52 names $(60 \%)$ are here represented by namebearing types ('primary types').

Specific and subspecific names are alphabetically entered, as is usual in type catalogs; each name is followed by the generic one (and subgeneric one if it was mentioned) as it was spelled in the original binomen, or by the generic and specific ones in the case of subspecies or 'va. rieties'; bibliographic citation follows, as well as an account of the types, if these were mentioned, starting with those deposited in this Museum. An account of the type specimens actually housed follows, with their label data. According to the International Code of Zoological Nomenclature ( $4^{\text {th }}$ edition, 1999), allotypes are not 'name-bearing types'. If the country is not mentioned, Argentina is meant; in every other case, the country is mentioned first. Some specimens bear a registration number, entered in the Entomological Division register, but numbers under 10000 corre- spond to the old general register of the museum. Later nomenclatural or taxonomical changes are added, as far as they are known to the author.

For the identification of types not formally designated in the original publication (art. 72.4 of the Code), evidences from the labels, as they are written in the collections examined by authors of names, were used. When the authors did not formally designate a holotype, or its equivalent, and did not mention the amount of specimens examined, it is assumed that they had a series of syntypes (recommendation $73 \mathrm{~F}$ of the Code), eventually 'unique syntypes'.

There are some specimens labelled as types, of names not found in the literature, and assumed not to have been published. These names are probably not available in the sense of the Code, but in order to assist in future research, they are listed separately.

Specimens designated as types after the original publication date, published or not, cannot be accepted as included in the type series, but, also in order to assist in future research, they are included in a further separate list along with other specimens labelled as types, but not included in the original publication.

This Museum is identified by MACN; repositories of other types are identified as follows: 
AOB, A.O. Bachmann coll., in MACN; BC, C. Berg Collection, in part in MACN; BM, The Natural History Museum, London; BRC, C. Bruch coll.; in part in MACN; CAS, California Academy of Sciences, S. Francisco; CISC, California Insect Survey, Berkeley; CNC, Canadian National Collection, Ottawa, Canada; EPCSI, Ecuador PeaceCorps - Smithsonian Instit. Aquatic Insect Surm vey; FIML, Fundación-Instituto Miguel Lillo, Tucumán, Argentina; FMNL, Field Museum of Natural History, Chicago, USA; INHS, Mllinois Natural History Survey, Urbana; JBC, Jean Brèthes Collection, in MACN; MCZ, Museum of Comparative Zoology, Cambridge, USA; MLP, Museo de La Plata, La Plata, Argentina; PC, M. Pic collection, Paris Museum; MZSP, Museu de Zoologia, São Paulo, Brasil; OC, R. Oberthür coll., Germany; RC, M.A. Régimbart coll., France; RIC, Richter coll., in MLP; TRC, E.R. Trémouilles coll., Buenos Aires; USNM, United States National Museum (Smithsonian Institution), Washington; ZMK, Zoologisk Museum, Köbenhavn, Danmark.

The classification given by Lawrence and Newton (1995) is here followed. Orychonotus excavatus Brèthes 1922 was identified by Reichardt in 1973 as a Limnichid, not a Nosodendrid, and therefore the entry was included here.

Fernández \& Cabrera (1996) published a catalog of the types of Dytiscoidea, Haliploidea, Gyrinoidea and Hydrophiloidea kept in the Museo de La Plata, which, along with the present one, include most of the types kept in Argentinian Institutions; a few are in the Fundación e Instituto Miguel Lillo, Tucumán.

\section{CATALOG OF THE TYPES}

\section{GYRINOIDEA: GYRINIDAE}

selindanus [Aulonogyrus caffer subsp.]. Brinck 1955: 341: Southern Rhodesia.

1 parat., pinned, "Chirinda For-/ est, S. Rhod. XII/-1937, G. van Son".

\section{HALIPLOIDEA: FALIPLIDAE}

bachmanni [Haliplus]. Vidal Sarmiento $\varnothing$ Grosso 1970: 65-67, pl. ii: f. 1-7: 2 o parat. from Chaco. Holot. $0^{*}$, allot. $\%, 10^{*}$ parat. from Formosa Prov: Ing. Juárez at MLP; 10 parat. from B. Aires Prov: Tigre [Paraná River delta], in AOB.

2 parat. 7725 on cards, Chaco, with a white paper disc " 3437 " (type register of MLP), with a glass microvial; 10 parat. B. Aires Prov.: Tigre, ex AOB. oblongus [Haliplus]. Zimmermann 1921: 182: 1 $0^{*}, 1 \%$ from B. Aires, in BRC.

1 synt. on a card, with a glass microvial, B. Aires Prov., labelled "Typus", ex BRC.

ornatipennis [Haliplus]. Zimmermann 1921; 182-183: 6 ex. from Tucumán, in BRC.

2 synt. (both?) on cards, Tucumán Prov, labelled "Typus" and "Cotypus" (printed on pale green paper) respectiv., the first one with an identification label handwritten by Zimmermann; ex BRC.

Note; from Zimmermann's paper it is not clear if he intended to designate a type; Bruch's printed labels suggest that there were name-bearing and non-name-bearing types; on the other hand Bruch's handwritten identification labels differ in shape and aspect, suggesting that he received the specimens at different times, and that the "Cotypus" (with the later label) could not have been included in the original series.

subseriatus [Haliplus, Haliplidae]. Zimmermann 1921: 181-182: 1 $\%$ from Tierra del Fuego.

Holot.? $O^{\pi}$ on a card, with a glass microvial, Tierra del Fuego, labelled "Typus", ex BRC.

Note: The locality agrees with, but the sex of the specimen differs from that mentioned by Zimmermann (a transcription error?).

\section{DYTISCOIDEA: DYTISCIDAE AND NOTERIDAE}

arauco [Lancetes, Dytiscidae] Bachmann \& Trémouilles 1981: 106, 113-115, f. 17: Holot. o from Mendoza, ex BRC. Allot. $q$ from Neuquen Prov.: La Pintada, at MLP; parat. in MLP, IFML and $\mathrm{AOB}$.

Holot. 0 , pinned, with a glass microvial, Mendoza, ex BRC; 1 parat. Neuquen Prov: La Pintada, ex AOB.

bifasciatus [[Bidessus] (Hemibidessus), Dytiscidael. Zimmermann 1921: 197, 206: 5 ex. from Brasil: Corumbá; 2 ex., somewhat larger, from Argentina: Santa $\mathrm{Fe}$, in BRC.

1 synt, on a card, S. Fe Prov, ex BRC.

Note: Recorded under Hemibidessus by Young (1969: 2).

bruchi [sub Bruchi] [Canthydrus, Noteridae]. Zimmermann 1919: 115-116: 2 ex. from B. Aires in BRC. Name of Régimbart in litt.

1 synt. on a card, labelled "Typus", ex BRC. 
Note: Recorded under Suphisellus by Bruch (1927: 538, 540).

bruchi [sub Bruchi]. [Derovatellus, Dytiscidae]. Zimmermann 1919: 125-126: 3 ex. from Buenos Aires, in BRC. Name of Régimbart in litt.

1 synt. on a card, B. Aires Prov, ex $\mathbf{B R C}$.

Note: Designated type species of Mesovatellus Trémouilles (1995: 25, 26-27),

calileguai [Rhantus, Dytiscidae]. Trémouilles 1984: 10, 17-18, f. 5, 23-29: Holot. $\sigma^{7}$ from Jujuy Prov.: Calilegua Nation. Park.

Holot. $0^{*}$, pinned, with a glass microvial, Jujuy Prov: Calilegua Nation. Park: Aguada del Tigre.

conicus [Bidessus (Hemibidessus), Dytiscidae]. Zimmermann 1921: 196-197, 206: Brasil: Corumbá, in large amounts; 1 ex. from Paraguay. Name of Régimbart in litt.

2 synt. on cards, on 1 pin, Brasil: Mato Grosso State: Corumbá, labelled "Cotypus", ex BRC.

Note: Recorded under Hemibidessus by Young (1969: 2).

dubius [Bidessonotus, Dytiscidae], Young 1990: 364-366, f. 10: Venezuela: Miranda, Guárico, Bolivar; Brasil: Mato Grosso: Jacaré; types at Florida State Coll. Arthrop., Gainesville, USA.

$10^{*}, 10$ parat. on pointed cards, the $0^{x}$ with dissected parts glued to a further pointed card, Venezuela: Guárico: $44 \mathrm{~km}$ S of Calabozo.

hydrovatoides LQueda, Dytiscidae]. Zimmermann 1921: 191-192: Brasil: Mato Grosso State: Corumbá; in large amounts.

2 synt. on cards, on 1 pin, Brasil: Mato Grosso State: Corumbá, labelled "Cotypus", ex BRC.

inexpectatus [Hydaticus (Guignotites), Dytiscidae]. Trémouilles 1996: 26, f. 8, 14, 25, 32, 40: Holot. $0^{*}$, allot. $₹, 23$ parat. from Ecuador: Los Ríos Prov.: Pichilingue.

Holot. $\sigma^{2}$, allot. 7,23 parat., all pinned, Ecuador: Los Ríos Prov.: Pichilingue.

knischi [sub Knischi] [Agaporomorphus, Dytiscidae]. Zimmermann 1921: 203-206: Brasil: Mato Grosso State: Corumbá, a numerous series.

2 synt. on cards, on 1 pin, Brasil: M. Grosso State: Corumbá, labelled "Cotypus", ex BRC.

magnus [Megadytes (Bifurcitus), Dytiscidae]. Trémouilles \& Bachmann 1981: 118-122, pl. vi: 10-18: Holot. 0 , allot. $\&$ from Santa Fe Prov.; Ceres; parat.: 10, 1 f from Santiago del Estero; $1 \%$ from $\mathrm{S}$. $\mathrm{Fe}$, in $\mathrm{BRC} ; 1 \sigma^{*}$ from $\mathrm{S}$. Fe Prov:
Ceres; $10^{\star}, 1 \% 15803$ from Salta Prov: Campo Durán; $10^{7}$ from Paraguay: Boquerón Dept.: Cerro León; $20^{\prime \prime}$ without locality label 2110,2111 . Other parat. from the same localities, and from Formosa Prov.: Ing. Juárez, and E. Ríos Prov.: Primero de Mayo, at MLP, AOB and TRC.

Holot. $O^{7}$ with a glass microvial, allot. \&, 3 parat. S. Fe Prov.: Ceres, 2 of them ex TRC; 3 parat. Santiago del Estero Prov., 1 of them 15803; 2 parat. Formosa Prov.: Ing. Juárez, ex $\mathbf{A O B}$; 1 parat. E. Ríos Prov.: Primero de Mayo, ex $\mathbf{A O B}$; 1 parat. Paraguay: Boquerón Departm.: Cerro León.

microscopicus [Bidessus, Dytiscidae]. Zimmermann 1921: 193, 206: Brasil: Mato Grosso State: Corumbá, the species seems to be abundant. 2 synt. on cards on 1 pin, Brasil: M. Grosso State: Corumbá, labelled "Cotypus", ex BRC.

Note: Recorded under Liodessus by Young (1969: 3).

nahueli [Rhantus antarcticus subsp., Dytiscidae]. Trémouilles 1984: 10, 18-19, f. $3,30,32,33,35$ : Holot. $0^{*}$, allot. ' $\$, 30^{*}, 15$ \& parat. from Río Negro Prov.: S. Carlos de Bariloche, 1 q parat. from R. Negro Prov: Puerto Pañuelo. Other parat. in MLP, IFML, ZMK, TRC and AOB.

Holot. $\sigma^{x}$ with a glass microvial, allot. $\%, 17$ parat., 2 of them with glass microvials, all pinned, R. Negro Prov.: Bariloche and Puerto Pañuelo.

parvus [Cybister, Dytiscidae]. Trémouilles 1985 : 187-188, f. 1-6: $10^{*}, 1$ \& parat. from Brasil: Goiás State: S. Rita. Holot. $0^{*}$, allot. $0,50^{*}, 3 \%$ parat., in MZSP; $10,1 \%$ parat. in MLP.

10 parat. (with a glass microvial), $1 \%$ parat., both pinned, Brasil: Goiás State: S. Rita.

pescheti [sub Pescheti] [Bidessus, Dytiscidae]. Zimmermann 1921: 194, 206: Brasil: Mato Grosso State: Corumbá, in very large amounts.

3 synt, on cards, on 1 pin, Brasil: Mato Grosso State: Corumbá, ex BRC.

Note: A synonym of Neobidessus alternatus (Régimbart 1889), fide Young (1969: 3).

rufulus [Suphisellus, Noteridae]. Zimmermann 1921: 188: Mato Grosso State: Corumbá; several dozen specimens.

2 synt. on 1 pin, Brasil: Mato Grosso State: Corumbá, labelled "Cotypus".

turbinatus [Hydrovatus, Dytiscidae]. Zimmermann 1921: 191: Buenos Aires, in BRC. Name of Régimbart in litt.

2 synt. (both?) on cards, B. Aires Prov., both 
labelled "Typus", 1 of them labelled by Zimmermann as "Hydrovatus turbinatus Rég. n. sp. typ.", and by Bruch as "Hydrovatus / turbinator/Zimmer."; the other one without identification label. Ex BRC.

Note: The second specimen, not labelled by Zimmermann, is perhaps not a syntype.

tuyuensis [Hydaticus, Dytiscidae]. Trémouilles 1996: 17, 30-31, f. 1, 2a,b, 4, 19, 27, 35, 43: Holot. $\sigma^{\pi}$, allot. $q, 2 \sigma^{*}, 1$ p parat. from Buenos Aires Prov.: Paraná River delta; $10^{7}$ parat, from B. Aires Prov.: S. Clemente del Tuyú; 1 o parat. from B. Aires Prov: Punta Médanos; 1 i parat. from B. Aires Prov.: Miramar; $20^{n}, 1 \%$ parat. from Uruguay: Rocha Dept.

Holot. $0^{7}$, allot. $\%, 14$ parat., all pinned (4 with glass microvials): 5 B. Aires Prov.: Paraná River delta, 1 B. Aires Prov: Médanos, 1 B. Aires Prov.: S. Clemente del Tuyú, 2 B. Aires Prov: Miramar; 1 B. Aires Prov: Playa Chapadmalal, 1 Uruguay: Montevideo, and 3 Uruguay: Rocha Dept.

variicollis [Suphisellus, Noteridae]. Zimmermann 1921: 187: 2 ex. from Santa Fe Prov. in BRC; 1 ex. from Brasil: Corumbá.

1 synt. on a card, S. Fe Prov, labelled "Typus", ex BRC.

villae [Anodocheilus, Dytiscidae]. Young 1974: 8* 9, pl. i: 2. Venezuela: Bolivar State.

1 parat. on a card, Venezuela: Guárico State:

S. Fernando.

Note: The locality differs from that given in the original description, but the labels seem authentic.

\section{BYRRHOIDEA: LIMNICHIDAE, ELMIDAE DRYOPIDAE AND HETEROCERIDAE}

acutus [Eulimnichus, Limnichidae]. Wooldridge 1979: 7-8, f. 7: Costa Rica: Las Canas, holot. $0^{7}$, allot. \&, parat. in USNM; parat. Colombia: Magdalena; Salvador: Ilopango; Guatemala: Río Sis; Honduras: Río Humuya etc.; México: Chiapas, S.L. Potosí, Tamazunchale, Tabisco, Vera Cruz, in USNM, CAS, CISC, INHS, MCZ, H.P. Brown coll. Canas.

1 c parat. on a pointed card, Costa Rica: Las

amazonica [Pagelmis, Elmidae]. Spangler 1981: 286-294, f. 1-17: Ecuador: Pastaza Prov.: Tzapino; Napo Prov.: Limoncocha. Parat. in MACN. Holot. $\sigma^{*}$, allot. $q$ and parat. in USNM; other parat. in several institutions.
$12 \sigma^{7}, q$ parat. on pointed cards, Ecuador: Pastaza Prov: Tzapino, blacklight trap.

annectens [Hydora, Elmidae], Spangler \& Brown 1981: 599-602, f. 1-5, 8: Neuquen Prov: Quilquihue; Río Negro Prov.: W of San Martín de los Andes; El Bolsón, and Norquinco [all these under "Chile" by errorl; Chile: Cautín, Llanquihue, Malleco, N̈ble, Osorno, and Talca Prov. Parat. in MACN. Holot. $\sigma^{\prime \prime}$, allot. $\&$ and parat. in USNM; other parat, in several institutions.

1 parat. on a pointed card, Chile [sic, Argentina is meantl: R. Negro Prov.: El Bolsón.

argentinus [Dryops (Pomatinus), Dryopidae]. Berg 1885: 223-224: Surroundings of Buenos Aires; type in BC.

Holot., pinned, B. Aires, labelled "Typus" (printed in red), and Dryops / argentinus / Berg 1885" (handwritten by Berg), ex BC.

Note: Recorded under Helichus (Zaitzev 1908: 296). Probably a species of Onopelmus Spangler 1980 (A.O. Bachmann).

breyeri [Limnichoderus, Limnichidae]. Pic 1930: 43: Buenos Aires Prov.: Tigre [Paraná River delta], in $\mathrm{BRC}$ and $\mathrm{PC}$.

1 paralectot. on a card, B. Aires: Tigre, labelled "Typus" (handwritten by Bruch on pale green paper), and "Limnichoderus / Breyeri n. sp." (handwritten, seemingly by $\mathrm{Pic}$ ), ex. BRC.

Note: A synonym of Eulimnichus plebeius Sharp 1902 fide Wooldridge (1978: 170), who designates lectot.

bruchi [Heterocerus; Heteroceridae]. Grouvelle 1906: 131: Buenos Aires Prov.: La Plata.

2 synt. on cards, B. Aires, labelled "Typus" and "Cotypus" respectively (printed on pale green paper), and "Heterocerus / Bruchi" and "Bruch" respectively (handwritten by Grouvelle); ex BRC.

Notes: Transferred to Efflagitatus by Trémouilles (1993: 24), who states that $E$. splendidus Pacheco 1965 is a synonym. Efflagitatus Pacheco 1965 is a synonym of Heterocerus Fabricius 1792, fide Mascagni (1993: 1).

cordubensis [Helichus, Dryopidae]. Berg 1885: 224: Córdoba Prov., at the Sierras, and at the sur. roundings of Córdoba city, in MACN and BC.

2 synt. on one card, labelled "Typus" (printed in red), "Cordoba" (printed on green paper), and "Hylichus / Erichs," (handwritten, seemingly by Berg). 
corrinae [Eulimnichus, Limnichidae]. Wooldridge 1979: 3: Venezuela: Guárico: $12 \mathrm{~km} \mathrm{~S}$ of Calabozo, holot. $\varnothing$, many parat. $\%$ in USNM; other parat. id. holot., Argentina: Tucumán; Brasil: Pará: Santarém; Rio Zinha, in CAS, MCZ, H.P. Brown coll., D.P. Wooldridge coll.

1 parat. on a pointed card, Venezuela: Guárico: Calabozo, blacklight trap.

excavatus [Orychonotus, Limnichidae?]. Brèthes 1922: 299-301: Buenos Aires 10.VIL.1908, under Nosodendridae.

1 synt. on a pointed card, B. Aires 10. VII.1908, with a square piece of red paper, labelled "type!" (handwritten by Brèthes). Ex JBC.

Notes: Brèthes did not mention how many specimens he had seen, seemingly only one (sole syntype?). The specimen is also labelled "Not Nosodendridae / ? Limnichidae / Reichardt det. $1973^{\prime \prime}$.

jordanorum [Elmoparnus, Dryopidae]. Spangler \& Perkins 1986: 359-364, f. 1-8: Holot. $\sigma^{*}$, allot. $\uparrow$, and many parat. from Guiana, in USNM.

2 parat. on pointed cards, Guiana: Mazaruni: Potaro Distr., Takutu Mountains.

jujuyensis [Heterocerus, Heteroceridae]. Trémouilles 1999: 103-105, f. 1-7: Holot. o , allot. \& , 14 parat. from Jujuy Prov.: Yala pond 22.III.1982 leg. E. Angrisano.

Holot. $C^{x}$, allot. $\%$ and 14 parat. on cards, on 16 pins, Jujuy: Yala pond 22.III.1982 leg. E. Angrisano.

langleyae [Eulimnichus, Limnichidae]. Wooldridge 1979: 3-4, f. 2: Ecuador: Napo: $18 \mathrm{~km}$ E of Lago Agrio, holot. $0^{*}$, allot. $\%$, many parat. in USNM; other parat. Ecuador: Napo, in USNM, D.P. Wooldridge coll.

1 parat. on a pointed card, Ecuador: Agrio Lake, at Aguarico River; blacklight trap.

latus [Phalacrichus, Limnichidae]. Wooldridge 1982: 382, 386, f. 5: Venezuela: Guárico and Barinas; Brasil: Pará; Colombia: Meta; Ecuador: Napo; Portuguesa Is. Holot. $0^{*}$, allot. $Q$, parat. in USNM; other parat. in MCZ.

1 parat. on a pointed card, Venezuela: Barinas: Yuca River.

levis [Physemus, Limnichidae]. Wooldridge 1984: 538 , f. 1: Venezuela: Barinas: E of El Cantón, holot. $\checkmark$, allot. $q$, many parat. in USNM; other parat. from Brasil: Goiás: Itapuranga; Jacaré: Xingu Nat. Park; Trinidad Is. in FMNH, MCZ and CNC.

1 pin, specimen missing on the pointed card, Venezuela: Barinas: $\mathrm{E}$ of El Cantón, blacklight trap.

magnus [Byrrhinus, Limnichidae]. Wooldridge 1987: 307, f. 3: Ecuador: Napo: Lago Agrio, holot. $o^{*}$, allot. $q$ in USNM; parat. id.; Puerto Napo, Puerto Nuevo, and Perú: Cuzco: Quince Mil, in CNC and EPCSI.

1 parat. on a pointed card, Ecuador: Napo Prov.: Puerto Nuevo, blacklight trap.

oblongus [Limnichoderes, Limnichidae], Brèthes 1922: 297-299, f. 9: Buenos Aires Prov.: Tigre [Paraná River delta] 20.IX. 1908.

1 synt. on a card, B. Aires: Tigre 10.IX.1908, labelled "type!" (handwritten by Brèthes), and with a square piece of red paper. $E x$ JBC.

Notes: Brèthes did not mention how many specimens he had seen, seemingly only one (sole syntype?). Recorded under Limnichoderus by Blackwelder (1944: 274).

plenus [Byrrhinus, Limnichidae]. Wooldridge 1987: 305-307, f. 2: Ecuador: Cotopaxi: Quevedo, holot. $\sigma^{x}$, allot. $q$ in USNM; parat, id. and Bolivia: Chuluriani [sic] (probably Chulumani), in $\mathrm{BM}, \mathrm{CNC}$ and EPCSI.

1 parat. on a pointed card, Ecuador: ENE of Quevedo, blacklight trap.

rudis [Phalacrichus, Limnichidae]. Wooldridge 1982: 382, 385-386, f. 4: Venezuela: Guárico; Suriname. Holot. $\%$, allot. $\%$, parat. in USNM; other parat. in FMNH.

1 parat. on a pointed card, Venezuela: Guárico: S of Calabozo, blacklight trap.

santacruzensis [Heterocerus, Heteroceridae]. Trémouilles 1999: 105-108, f. 8-13: Bolivia: Santa Cruz: El Cidral 1-28.1.1962 leg. R. Golbach. 20 parat. Holot. $\sigma^{x}$, allot. $q$ and 10 parat. in FIML.

10 parat. on cards, on 5 pins, Bolivia: El Cidral 1-28.I.1962 leg. R. Golbach.

simplex [Phalacrichus, Limnichidae]. Wooldridge 1982: 383, 387-388, f. 8: Venezuela: Guárico; Bolivia: Beni; Brasil: Goiás, Mato Grosso; Colombia: Meta. Holot. $\sigma^{*}$, allot. $\%$, parat. in Mus. Washington; other parat. in FMNH and MCZ.

1 parat. on a pointed card, Venezuela: Guárico, S of Calabozo, blacklight trap.

spangleri [Phalacrichus, Limnichidae]. 
Wooldridge 1982: 383, 386-387, f. 6: Venezuela: Barinas, Zulia; Ecuador: Napo. Holot. $0^{*}$, allot. $\%$, parat. in USNM.

1 parat. on a pointed card, Venezuela: Barinas: E of El Cantón, blacklight trap.

subantarcticus [Heterocerus, Heteroceridae]. Trémouilles 1999: 108, f. 14-19: Holot. $\sigma^{\circ}$ and 1 parat. $\sigma^{7}$ from Chile: Punta Arenas 10.IV.1961 leg. T. Cekalovic.

Holot. $O$ and 1 parat. $\sigma^{*}$ on cards, on 1 pin, Chile: Punta Arenas 10.IV.1961, leg. T. Cekalovic.

temporalis [Martinius, Limnichidae]. Wooldridge 1988: 315-315, f. 1, 2: Ecuador: Manabi: Bahia de Caraquez, holot. $\sigma^{\prime}$, allot. $\%$, many parat. in USNM.

1 parat. on a pointed card, Ecuador: Caraquez Bay, brackish lagoon.

validus [Heterocerus, Heteroceridae]. Grouvelle 1906: 131-132: Buenos Aires Prov.

1 synt. on a card, B. Aires, labelled "Typus" (printed on pale green paper), and "Heterocerus / validus / Grouv." (handwritten by Bruch), ex BRC.

Note: Transferred to Efflagitatus by Pacheco (1965: 121); Efflagitatus is a synonym of Heterocerus Fabricius 1792, fide Mascagni 1993: 1.

\section{SCIRTOIDEA: SCIRTIDAE}

bruchi [Ora]. Pic 1928: 8-9; Argentina, in BRC and $\mathbf{P C}$.

1 synt. on a card, Buenos Aires Prov., labelled "Typus" (handwritten by Bruch on pale green paper), and "Ora Bruchi n. sp." (handwritten, seemingly by $\mathrm{Pic}$ ).

diversenotatus [Scirtes adustus, var.]. Pic 1930: 43-44. Córdoba Prov: San Javier: La Paz, in BRC.

1 synt. on a card, Córdoba: La Paz, labelled "Typus" (handwritten by Bruch on pale green paper), and "Scirtes/ confusus [sic] v. nov. diversenotatus" (handwritten, seemingly by $\mathrm{Pic}$ ), ex BRC.

Note: Pic did not mention how many specimens he had seen, seemingly only one (sole syntype?).

platensis [Ora]. Brèthes 1925: 13: Buenos Aires 4.II. 1922, at electric light.

1 synt. on a card, B. Aires 24.III.1923, labelled "type!" (handwritten by Brèthes), and with a square piece of red paper. $E x$ JBC.

Note: Brèthes did not mention how many specimens he had seen; the date differs from the published one.

\section{NAMES NOT FOUND IN THE BIBLIOGRAPHY}

The following names were not found in the bibliography, and were probably not published.

\section{brevenotata [Ora, Scirtidae]. Pic}

1 ex. on a card, Chaco Prov., labelled "Typus" (handwritten by Bruch on pale green paper), and "Ora brevenotata Pic var." (handwritten, seemingly by Pic), ex BRC.

bruchi [Helichus, Dryopidae]. Grouvelle.

1 ex. on a card, Misiones Prov., labelled "Typus", and "Helichus Bruchi Grouv." (handwritten by Grouvelle), ex BRC.

bruchi [Pelonomis, Dryopidae], Grouvelle.

3 ex. on eards, 1 of them Córdoba Prov., labelled "Typus" (printed on pale green paper), and "Pelonom. Bruchi" (handwritten by Grouvelle); 2 of them Tucumán Prov., labelled "Cotypus" (printed on pale green paper), ex BRC.

crassipes [Helichus, Dryopidae]. Grouvelle.

1 ex. on a card, from "Corumbá-Paraguay" (sic), labelled "Cotypus" (printed on pale green paper), and "Helic. crassip." (handwritten by Grouvelle), ex BRC.

dubitabilis [Pelonomus, Dryopidae] Grouvelle.

1 ex. on a card, Tucumán Prov, labelled "Cotypus" (printed on pale green paper), and "Pelonomus dubitabilis" (handwritten by Grouvelle), ex BRC.

elongatus [Pelonomus, Dryopidae]. Grouvelle.

1 ex. on a card, Buenos Aires Prov, labelled "Typus" (printed on pale green paper), and "Pelonom. elongat." (handwritten by Grouvelle), ex BRC.

iheringi [Laccophilus, Dytiscidae]. Régimbart.

2 ex. on cards, Brasil: S. Paulo, labelled "Cotypus", ex BRC.

sulcicollis [Dryops, Dryopidae]. Grouvelle

1 ex. on a card, Buenos Aires Prov., labelled "Typus" (printed on pale green paper), and "Dryops / sulcicollis / Grouv." (handwritten by Bruch), ex BRC. 


\section{SPECIMENS LABELLED AS TYPES BUT NOT INCLUDED IN THE ORIGINAL SERIES}

angustus [Laccophilus, Dytiscidae]. Régimbart 1889: 266: Paraguay: Asunción, and Brasil: Mato Grosso State, in OC and RC.

1 ex., Brasil: S. Paulo, labelled "Cotypus", det. Régimbart, ex coll. Bruch.

Note: S. Paulo was not mentioned in the original publication.

bergi [Heterocerus, Heteroceridae], Grouvelle 1906: 132-133: Buenos Aires Prov.

3 ex. on cards, on 2 pins, with a glass microvial each, Tucumán Prov, labelled "Typus" ( 1 ex.) and "Cotypus" (2 ex.), the first one, in addition, "Heterocerus / Bergi / Grouv. typ." (handwritten by Bruch), and "H. bergi / Grouvelle det." (handwritten by Grouvelle); ex BRC.

Notes: Tucumán was not mentioned in the original publication. Transferred to Tropicus by Trémouilles (1993: 24), who states that $T$. imperator Pacheco 1.965 is a synonym.

bergi [Pelonomus, Dryopidae]. Grouvelle 1896: 67: Uruguay, in MACN and GM.

3 ex. on cards, 2 of them Tucumán Prov, 1 of them Jujuy Prov., labelled "Cotypus" (printed on pale green paper), the 2 one labelled "Pelonomus / Bergi Grouv." (handwritten by Grouvelle).

Note: Tucumán and Jujuy were not mentioned in the original publication. Furthermore, the specimens were collected after the publication date.

flavolineatus [Canthydrus, Noteridae]. Régimbart 1889: 262-263: Brasil: Caraça, in OC and $\mathbf{R C}$.

1 ex., pinned, Brasil: S. Paulo, labelled "Cotypus", ex BRC.

Notes: S. Paulo was not mentioned in the original publication. Recorded under Suphisellus by Grosso (1979: 11, 88, f. 90, 91, 99, 128-131).

gigas [Pomatinus, Dryopidae]. Grouvelle 1897: 216: Brasil: Santa Catarina, in GC.

2 ex. on cards, labelled "Rep. Argentina/Prov. Buenos Aires / 7.XI.1899/C. Bruch" (printed, the date handwritten by Bruch), and "Typus" and "Cotypus" respectively (printed on pale green paper), ex BRC.

Notes: B. Aires was not mentioned in the original publication; furthermore, the specimens were collected after the publication date. Recorded as Helichus gigas by Blackwelder (1944: 273). obliquatus [Laccophilus, Dytiscidae]. Régimbart 1889: 264-265: Central Paraguay, and Brasil: Mato Grosso State, in $\mathbf{O C}$ and $\mathbf{R C}$; Oberthür says it is perhaps the same as $L$, ovatus.

1 ex. on a card, Buenos Aires, ex BRC.

Note: Buenos Aires was not mentioned in the original publication.

parallelus [Parnus, Dryopidae]. Grouvelle 1890: cxlvi: Brasil: Theresopolis.

1 ex. on a card, Buenos Aires Prov., labelled "Typus" (printed on pale green paper) and "parallelus Grouv." (handwritten by Grouvelle), ex BRC.

Notes: Buenos Aires was not mentioned in the original publication; furthermore, the specimen was collected after the publication date. Recorded and redescribed under Parygrus by Grouvelle (1896: 215-216).

piceonotatus [Cyphon liggeti, var, Scirtidae] [sub Liggetil. Pic:1918: 24: Chile.

1 ex. or a eard, Buenos Aires Prov., labelled "Typus" (handwritten by Bruch on pale green paper), and "Cyphon/Siggeti [sic]/v. piceonotatus Pic" (handwritten, seemingly by Pic).

Note: B. Aires was not mentioned in the original publication.

reticulatus [Notomicrus, Noteridae]. Zimmermann 1921: 188-189: 1 ex. from Santa Fe, in BRC. Name of Régimbart in litt.

1. ex., B. Aires Prov., ex RIC, labelled "Notomicrus / reticulatus / Rég, type" handwritten by Régimbart.

Note: The label seems authentic, but localities differ (mislabelled?).

\section{BIBLIOGRAFIA}

Bachmann, A.O. \& E.R. Trémouilles. 1981. El género Lancetes en la Argentina continental (Coleoptera, Dytiscidae). Physis, B. Aires, Sección B 39 (97): 103 $118, f .1-25$.

Berg, C. 1885. Quindecim Coleoptera nova faunae Reipublicae Argentinae. Anls. Soc. cient. argent. 19: 219-235.

Blackwelder, R.E. 1944. Checklist of the Coleopterous insects of Mexico, Central America, the West Indies, and South America (part 2), Bull., U. S. nation. Mus. $185(2): 189-341$.

Brèthes, 3 . 1922. Descripción de varios coleópteros de Buenos Aires. Anls. Soc. cient argent. 94: 263-307, 9 f.

-1925. Nouveaux coléoptères sudamericains. Nunquam otiosus, Buenos Aires $4(2)$ : 11-16. 
Brinck, P. 1955. Gyrinidae. In Hanström, B., P. Brinck \& G. Rudebeck (Ed.), South African Animal Life. Results Lund Univ. Exped. 1950-1951, Stockholm 1: iii +518 p.

Bruch, C. 1927. Catálogo sistemático de los coleópteros de la República Argentina. Suplemento II. Physis, B. Aires 8 (31): 536-553.

Fernández, L.A. \& N.C. Cabrera. 1996. Los ejemplares tipo de Dytiscoidea, Haliploidea, Gyrinoidea e Hydrophiloidea depositados en la colección del Museo de La Plata. Revla. Mus. La Plata, Ser técn. didáct. (24): 1-10.

Grosso, L.E. 1979. Contribución al conocimiento biológico y sistemático de las especies argentinas de Noteridae (Coleoptera, Adephaga). Tesis 374, Univ. Nac. La Plata: 166 p.

Grouvelle, A. 1890. Deux espèces nouvelles de parnides. Annls. Soc. entom. France, $6^{\circ}$ Sér. 10 (Bull.): cxlvi.

- 1896. Note sur le Pelonomus pubescens Blanch. et simplex Berg el description de quelques Dryopides et Helmides nouveaux. Anls. Mus. Hist. nat. B. Aires 5: 5-10.

- 1897. Nitidulides, Colydiides, Cucujides et Parnides recolectés par M. E. Grouvelle au Brésil et autres clavicornes nouveaux d'Amérique. Annls. Soc. entom. France 65, 1896; 177-216.

-1 1906. Quelques clavicornes nouveaux de la République Argentine recueillis par M. Charles Bruch. Revta. Mus. La Plata 12, 1905: 121-133.

International Commission on Zoological Nomenclature. 1999. International Code of Zoological Nomenclature, $4^{\text {th }}$ ed.: $\mathrm{xxix}+306 \mathrm{p}$.

Lawrence, J.F. \& A.F.N. Newton. 1995. Families and subfamilies of Coleoptera (with selected genera, notes, references and data on family group names. In Papaluk, I. \& S.A. - lipi ski (Ed.) Biology, phylogeny, and classification of Coleoptera. Papers celebrating the $80^{\text {th }}$ birthday of Roy $A$. Crowson, 1 , 2: ix $+1092 \mathrm{p}$.

Mascagni, A. 1993. La collezione eteroceridologica del Carnegie Museum of Natural History di Pittsburgh (U.S.A.), con descrizione di quattro nuove specie (Coleoptera: Heteroceridae). Opusc. zool. fluminensia $103: 1-12$, $\mathrm{C}$.

Pacheco, F.M. 1965. Sistemática, filogenia y distribución de los heterocéridos de América (Coleoptera: Heteroceridae). Esc. nac. Agric., Colegio Post-Grad., México, Chapingo. Monografia (1), 1964: 1-155, $501 \mathrm{f}$.

Pic, M. 1918. Nouveautés diverses. Mélanges Exot.entom. 29: 1 -24.

-1928. Notes et descriptions. Mélanges Exot.nenlom. 51: $1-36$.

-1930. Nouveaux coléoptères de diverses familles. Revta. Soc. entom, argent. 3 (1): 43-46.

Régimbart, M. 1889. Énumération des Haliplidae, Dytiscidae et Gyrinidae recueillis par $M$. le Professeur L. Balzan dans l'Amérique méridionale et description de quelques autres espèces voisines. Annli. Mus. civ. Stor, nat, Genova, Italy 27 ( $2 \mathrm{~d}$ Ser. 7): $256-268$.

Spangler, P.J. 1980. Onopelmus, a new genus of dryopid beetle from Peru (Coleoptera: Dryopidae). Proc. entom. Soc. Washington $82(2): 161-165$, f. 1-7.

- 1981. Pagelmis amazonica, a new genus and species of water beetle from Ecuador (Coleoptera: Elmidae). Pan-Pacif. Entom.: 286-294, f: 1-17.

Spangler, P.J. \& H.P. Brown. 1981. The discovery of Hydora, a hitherto Australian-New Zealand genus of riffle beetles, in austral South America (Coleoptera: Elmidae). Proc. entom. Soc. Washington $83(4)$ : 596-606, f. 1-8, tab. 1 .

Trémouilles, E.R. 1984. El género Rhantus Dejean en la Argentina (Coleoptera, Dytiscidae). Physis, B. Aires, Sección B 42 (102): 9-24, f. 1-46.

- 1985. Notas sobre Coleoptera acuáticos neotropicales. I. Cybister (Meganectes) parvus sp. nov. del Brasil (Coleoptera, Dytiscidae). Revta. Soc. entom. argent. $43: 187 \times 190$, f. 1-6.

-1 1993. La identidad de Heterocerus bergi Grouvelle 1905 y Heterocerus bruchi Grouvelle 1905 (Coleoptera, Heteroceridae). Revta. Soc. entom. argent. 50, 1991: 24 .

- 1995. Insecta Coleoptera Dytiscidae: Methlinae . Hydroporinae. In Castellanos, Z.A. de (dir) Fauna Agua dulce Rep. Argent. 37 (1):5-82, pl. i-xiv.

- 1996. Revisión del género Hydalicus Leach en América del Sưr con descripción de tres nuevas especies (Coleoptera, Dytiscidae). Physis, B. Aires, Sección B-52 (122-123), 1994: 15-32, f. 1-44.

- 1999. Deseripción de tres nuevas especies de Heterocerus Fabricius, de América del Sur (Coleoptera, Heteroceridae). Revta. Mus. argent. Cienc. nat., nueva Serie 1. (1): 103-108, f. 1-19.

Trémouilles, E.R. \& A.O. Bachmann. 1981. La tribu Cybisterini en la Argentina (Coleoptera, Dytiscidae). Revta Soc. entom. argent. 39 (1-2), 1980: 101.125, pl. i-vi.

Vidal Sarmiento, J. \& L.E. Grosso. 1970. Notas sobre halíplidos argentinos. I. (Coleoptera), Revta. Soc. entom. argent. 32: 63-67, pl, i-ii.

Wooldridge, D.P. 1978. New World Limnichinae. IV. Eulimnichus Casey. A. Synonymies, lectotype designations and redescriptions (Coleoptera: Limnichidae). Great Lakes Entom. 11 (3): 163-173, 11 f.

-1979. New World Limnichinae. IV. Eulimnichus Casey. B. Descriptions of new species (Coleoptera: Limnichidae). Great Lakes Entom. 12 (1): 1-11, f.1-9

- 1982. New World Limnichinae. VII. Phalacrichus Sharp. A recharacterization and eight new species (Coleoptera: Dryopoidea: Limnichidae). Coleopt.'s Bull. 36 (2): 381-389, f. 1-11.

- 1984. Two new species of Physemus LeConte from South America (Coleoptera: Dryopoidea: Limnichidae). J. Kansas entom. Soc. 57 (3): 538 539.

- 1987. New World Limnichinae 9: a revision of Neotropical Byrrhinus Motschulsky (Coleoptera: Dryopoidea: Limnichidae). Coleopt.'s Bull. 41 (4): 303.314 ,

- 1988. Martinius temporalis, a new species from Ecuador (Coleoptera: Limnichidae: Thaumastodinae). J. New York entom. Soc. 96: 314. 315, f. $1,2$.

Young, FN. 1969. A check-list of the American Bidessini (Coleoptera: Dytiscidae: Hydroporinae). Smithson. 
Contr, Zool, Washington, USA (33): 1-5.

-. 1974. Review of the predaceous water beetles of genus Anodocheilus (Coleoptera: Dytiscidae: Hydroporinae). Occas. Pap. Mus. Zool., Univ. Michigan (670): 1-28, $3 \mathrm{pl}$.

-1990. Review of classification of the water beetles of the New World genus Bidessonotus Régimbart (Coleoptera: Dytiscidae: Hydroporinae: Bidessini). Quaest. entom. 26 (3): 355-381.
Zaitzev, P. 1908. Catalogue des Coléoptères aquatiques des familles des Dryopidae, Georyssidae, Cyathoceridae, Heteroceridae et Hydrophilidae. Horae Soc. entom. ross. 38: 283-420.

Zimmermann, A. 1919. Schwimmkäfer des Deutschen Entomologischen Museums in Berlin-Dahlem. Arch. Naturgesch., Berlin A 83 (13), 1917: 68-249, $21 \mathrm{f}, 2$ tab.

- 1921. Beiträge zur Kenntnis der südamerikanischen Schwimmkäferfauna, nebst 41 Neubeschreibungen. Arch. Naturgesch., Berlin A 87 (3), 1920: 181.206.

Recibido: 9-IX-2002

Aceptado:4-X1-2002 